\title{
A Model of Cancer Clinical Trial Decision-making Informed by African-American Cancer Patients
}

\author{
Jennifer A. Wenzel • Olive Mbah • Jiayun Xu • \\ Gyasi Moscou-Jackson • Haneefa Saleem • Kwame Sakyi • \\ Jean G. Ford
}

Received: 4 August 2014 /Revised: 19 September 2014 / Accepted: 6 October 2014 /Published online: 23 October 2014

(C) W. Montague Cobb-NMA Health Institute 2014

\begin{abstract}
Background Clinical trials are critical to advancing cancer treatment. Minority populations are underrepresented among trial participants, and there is limited understanding of their decision-making process and key determinants of decision outcomes regarding trial participation.

Methods To understand research decision-making among clinical trial-eligible African-American cancer patients at Johns Hopkins, we conducted seven focus groups $(n=32)$ with trial-offered patients $\geq 18$ years diagnosed with lung, breast, prostate, or colorectal cancer $\leq 5$ years. Three "acceptor" and four "decliner" focus groups were conducted. Questions addressed: attitudes towards clinical trials, reasons for accepting or declining participation, and recommendations to improve minority recruitment and enrollment. Data were transcribed and analyzed using traditional approaches to content and thematic analysis in NVivo 9.0. Data coding resulted in themes that supported model construction.

Results Participant experiences revealed the following themes when describing the decision-making process: Information gathering, Intrapersonal perspectives, and Interpersonal influences. Decision outcomes included the presence or absence of decision regret and satisfaction. From these themes, we generated a Model of Cancer Clinical Trial Decision-making.
\end{abstract}

J. A. Wenzel $(\bowtie) \cdot J . X u \cdot G$. Moscou-Jackson

Johns Hopkins School of Nursing, 525 N. Wolfe St, Baltimore, MD 21205-2110, USA

e-mail: jwenzel@jhu.edu

O. Mbah $\cdot$ H. Saleem $\cdot$ K. Sakyi $\cdot$ J. G. Ford

Bloomberg School of Public Health, Baltimore, MD, USA

Present Address:

J. G. Ford

Department of Medicine, The Brooklyn Hospital Center, Brooklyn, NY, USA
Conclusion Our model should be tested in hypothesis-driven research to elucidate factors and processes influencing decision balance and outcomes of trial-related decision-making. The model should also be tested in other disparities populations and for diagnoses other than cancer.

Keywords Clinical trials $\cdot$ African-Americans $\cdot$ Decision balance $\cdot$ Decision regret $\cdot$ Cancer disparities

\section{Introduction}

Racial and ethnic minority populations in the USA continue to experience disproportionately higher cancer mortality rates [1], lower survival rates, and poorer quality of cancer treatment $[2,3]$. African-Americans have the lowest 5-year survival rate for nearly all cancer sites [1]. Clinical trials (CTs) are a key step in the development of new cancer therapies, and access to CTs may reduce cancer health disparities [4-7]. Trial participants are usually monitored closely and may have better treatment outcomes than non-participants [8-10]. Yet, only $3-$ $5 \%$ of adult cancer patients participate in CTs, and among them, fewer than $10 \%$ are racial and ethnic minorities [11]. African-American men are especially unlikely to enroll [12].

This underrepresentation limits the ability to generate hypotheses about differences in outcomes or to conduct meaningful subgroup analyses in order to detect between-group differences in response to treatment. This results in unfair distribution of the potential benefits and risks of trial participation and decreases generalizability of research findings [13]. Numerous factors contribute to decreased clinical trial participation among racial and ethnic minorities. Among these factors, medical distrust is the most frequently reported barrier $[14,15,16]$. Despite knowledge of the barriers to participation, there is scant information on the process of trial participation decision-making among African-American cancer 
patients. Among cancer patients who have been offered trial participation, key factors in the decision-making process and determinants of trial decision outcomes remain incompletely understood, when comparing those who have accepted participation (acceptors) and those who have declined (decliners).

The purpose of this study was to examine the processes and motivations of African-American cancer patients at the Johns Hopkins Sidney Kimmel Comprehensive Cancer Center (JHSKCCC) for accepting or declining participation in cancer trials, and to elucidate the outcomes of these decisions. The study was conducted under the auspices of Enhancing $M$ inority Participation in Clinical Trials (EMPaCT), a consortium of five comprehensive cancer centers, whose goal is to create and develop evidence-based strategies to enhance trial participation among underrepresented minorities. We used qualitative methods to discover underlying factors, processes, and outcomes related to the complex phenomenon of trial decision-making [17-19]. Based on the data and extant literature, we developed the Model of Cancer Clinical Trial Decision-making among African-American cancer patients.

\section{Patients and Methods}

In 2011, we conducted seven focus groups (32 patients $=14$ acceptors; 18 decliners) with African-American patients who met the following eligibility criteria: (1) age $\geq 18$ years; (2) diagnosed with lung, breast, prostate, or colorectal cancer $\leq 5$ years, (3) received cancer treatment at JH-SKCCC, and (4) offered CT participation. Based on literature recommendations, we held separate groups for those who had agreed to participate in a trial $(n=3)$ and those who declined $(n=4)$, with 4-5 participants per group [20-22]. Patients were recruited through JH-SKCCC database reviews and clinic staff referrals available via a HIPAA waiver. In a letter signed by $\mathrm{JH}-$ SKCCC Director and our EMPaCT Principal Investigator, potential participants were invited to take part in the study or to opt out. Study staff then telephone-screened patients. Of the 187 patients screened for the study, 64 patients were eligible and invited to participate.

Because information on which patients are offered trial participation was not routinely reported or accessible across JH-SKCCC databases during the recruitment period, we used patient self-report to identify participants [23-25]. Information on patients who declined to participate when offered our study was collected over the phone during the screening eligibility process. Thus, we were able to compare them to study participants on demographics (such as name, date of birth), cancer diagnosis and treatment status, as well as clinical trial participation.

Participants provided written informed consent and completed a demographic questionnaire (age, gender, socioeconomic status, comorbid conditions, cancer diagnosis-related information) prior to the focus group interviews. We used a semi-structured discussion guide developed through team discussion, with minor refinements based on feedback from initial focus groups. The guide addressed the following topics: experiences with cancer diagnosis, attitudes towards trials, decision-making processes, reasons for participation/non-participation, trial barriers, and recommendations to improve trial participation. Sample questions included: "How did you make the decision to [participate/not participate]?" "What do you think African American patients and the people who care about them need to know about participating?"

An experienced facilitator/observer team with no clinical JH-SKCCC roles moderated all groups. Each group had at least one racially concordant facilitator. Groups lasted $\sim 90$ min, were digitally recorded, and professionally transcribed verbatim. Participants received US\$50 and transportation compensation. The study was approved by the Johns Hopkins School of Public Health Institutional Review Board.

\section{Data Analysis}

We conducted qualitative content and thematic analysis of the transcripts [26], using NVivo 9.0. At least two coders reviewed each transcript, using a previously described inductive approach to coding [27]. The team developed all concepts and themes, based on the final codes. The coding team $(n=4)$ met regularly to refine code definitions, discuss alternative interpretations, and select representative quotations for final review. Inter-rater reliability (kappa) among coders for all transcripts was $>0.8$. Study meetings were regularly convened to review findings and incorporate them into the model. Team members' recommendations were incorporated into the final model.

\section{Results}

Nearly $50 \%$ of patients who were screened and eligible participated in focus groups held at a local community-based primary care clinic. Participants were similar to nonparticipants with respect to age and gender. Overall, there were no clear differences in sociodemographic factors between acceptors and decliners or by gender except that acceptors were more likely to report incomes of $\leq \mathrm{US} \$ 35,000$ (Table 1). On average, male participants were older and were less likely than female participants to report incomes of $<$ US\$35,000 (Table 2). Nearly all had completed active treatment prior to study enrollment: $7 / 32$ patients were $<1$ year from diagnosis, 14 within $1-2$ years, 5 within 2-3 years, and 7 within 3-5 years of diagnosis. Among similar trials conducted at JH-SKCCC within our study timeframe (2008-2012), only data on trial acceptors were available. Our study population 
Table 1 Demographic characteristics of study population by clinical trial status $(n=32)$

\begin{tabular}{|c|c|c|c|}
\hline Demographic & $\begin{array}{l}\text { Acceptors, } n \\
(\%)^{*}\end{array}$ & $\begin{array}{l}\text { Decliners, } n \\
(\%)^{*}\end{array}$ & $\begin{array}{l}\text { Total } \\
n^{*}\end{array}$ \\
\hline Age (years): median [range] & $58[32-77]$ & $55[41-73]$ & \\
\hline \multicolumn{4}{|l|}{ Gender } \\
\hline Male & $6(42.9)$ & $6(33.3)$ & 12 \\
\hline Female & $8(57.1)$ & $12(66.7)$ & 20 \\
\hline \multicolumn{4}{|l|}{ Education } \\
\hline$\leq$ HS/technical school & $8(57.1)$ & $8(44.4)$ & 16 \\
\hline$>$ Some college & $6(43.9)$ & $10(55.6)$ & 16 \\
\hline \multicolumn{4}{|l|}{ Income } \\
\hline$<\mathrm{US} \$ 35,000$ & $10(76.9)$ & $7(43.8)$ & 17 \\
\hline$\geq \mathrm{US} \$ 35,000$ & $3(23.1)$ & $9(56.2)$ & 12 \\
\hline \multicolumn{4}{|l|}{ Cancer type } \\
\hline Breast & $8(57.1)$ & $12(66.7)$ & 20 \\
\hline Prostate & $4(28.6)$ & $5(27.8)$ & 9 \\
\hline Colorectal & $1(7.1)$ & $1(5.5)$ & 2 \\
\hline Lung & $1(7.1)$ & 0 & 1 \\
\hline \multicolumn{4}{|l|}{ Comorbidities } \\
\hline 0 & $2(14.3)$ & $3(16.7)$ & 5 \\
\hline $1-2$ & $10(71.4)$ & $10(55.5)$ & 20 \\
\hline $3-4$ & $2(14.3)$ & $5(27.8)$ & 7 \\
\hline Time since Dx, median (years) & 1.4 & 1.8 & \\
\hline
\end{tabular}

*Except for age, time since Dx

resembled African-American cancer center patient acceptors in median age (58 in our sample; 57 in the JH-SKCCC); however, compared to cancer center patients enrolled in trials, our participants were more likely to be female $(57 \%$ in our sample; $49 \%$ in the JH-SKCCC).

Data revealed multiple themes/subthemes (Table 3) related to trial decision-making. These were consistent among both acceptors and decliners. Participants described a process of decision balance: weighing information, influences, and options to arrive at the decision to participate or not. Participants' information gathering, as well as intrapersonal and interpersonal influences, is a key component of decision processing. Trial decision-making was presented as either balanced or imbalanced and influenced decision outcomes, namely, satisfaction with decision-making and the presence or absence of decision-related regret. Some participants verbalized regret over what they perceived as a lost opportunity to convert the difficulties of their cancer experience into a communal good. Some decliners also expressed regret when they allowed decision partners to sway them against trial participation. Racial/ethnic disparities served as an implicit background for decision-making. Some participants described experiencing a health disparities dilemma: mistrust in research juxtaposed against a perceived obligation to participate, as a way to contribute to knowledge gains that may reduce treatment
Table 2 Demographic characteristics of study population by gender $(n=$ 32)

\begin{tabular}{llll}
\hline Demographic & Male, $n(\%)^{*}$ & Female, $n(\%)^{*}$ & $\begin{array}{l}\text { Total } \\
n^{*}\end{array}$ \\
\hline $\begin{array}{l}\text { Age (years): median [range] } \\
\text { Education }\end{array}$ & $67[49-77]$ & $50[32-69]$ & \\
$\quad \leq$ HS/technical school & $7(58.3)$ & $9(45.0)$ & 16 \\
$\quad>$ Some college & $5(41.7)$ & $11(55.0)$ & 16 \\
Income & & & \\
$\quad<$ US $\$ 35,000$ & $5(45.4)$ & $12(66.7)$ & 17 \\
$\geq$ US\$35,000 & $6(54.5)$ & $6(33.3)$ & 12 \\
Cancer type & & & \\
Breast & 0 & $20(100)$ & 20 \\
Prostate & $9(75.0)$ & 0 & 9 \\
Colorectal & $2(16.7)$ & 0 & 2 \\
$\quad$ Lung & $1(8.3)$ & 0 & 1 \\
Clinical trial status & & & \\
Acceptors & $6(50.0) 6(50.0)$ & $8(40.0)$ & 14 \\
Decliners & & $12(60.0)$ & 18 \\
Comorbidities & & & \\
0 & $2(16.7)$ & $3(15.0)$ & 5 \\
1-2 & $8(66.6)$ & $12(60.0)$ & 20 \\
3-4 & $2(16.7)$ & $5(25.0)$ & 7 \\
Time since Dx, median (years) & 1.7 & 1.3 & \\
\hline
\end{tabular}

*Except for age, time since Dx

disparities. Health disparities dilemma arose as participants' compared and contrasted participation with feelings of altruism in the face of research mistrust. Many participants expressed a personal responsibility or internal burden to participate in trials to ameliorate health and treatment disparities. However, the same participants who felt a personal responsibility to participate mentioned their mistrust in research within the same focus group discussion. Overall, achieving decision balance was a complex process encompassing perceived roles, responsibilities, research mistrust, and obligations to science or to family/friends.

We have modeled the themes and presented fuller explanations and exemplars for each theme/subtheme to accompany the model (Table 3). Participant-described barriers to trial participation were integrated into our model. Most have also been previously identified in the literature [15].

\section{Discussion}

In this study, we sought to understand the decision-making process and motivations of African-American cancer patients when making decisions about clinical trial participation. Using data from both acceptors and decliners, we were able to develop a focus group-derived Model of Cancer Clinical 
Table 3 Themes from Model: Descriptions and exemplars

\begin{tabular}{|c|c|c|}
\hline Themes/subthemes & Description & Selected exemplar data (individual groups are de-identified) \\
\hline $\begin{array}{l}\text { Theme: information } \\
\text { gathering }\end{array}$ & $\begin{array}{l}\text { Many factors influenced the decision-making process and } \\
\text { information gathering related to trials. Similar to other } \\
\text { literature reports, our participants discussed perceived } \\
\text { benefits and risks, understanding of trials, alternative } \\
\text { proven therapies, financial feasibility, communication, and } \\
\text { perceived conflicts of interest from providers as factors } \\
\text { which influenced trial participation decisions [26]. } \\
\text { Participants desired trial information from multiple sources: } \\
\text { different levels of care providers, current and former trial } \\
\text { participants, and existing support networks. } \\
\text { For study participants, being informed about a clinical trial } \\
\text { was important for them to be able to fully understand, feel } \\
\text { comfortable with, and make decisions about clinical trials } \\
\text { [14]. Participants wanted to be offered clinical trial }\end{array}$ & $\begin{array}{l}\text { "I have a concept of what I think a clinical trial is. But I think a } \\
\text { lot of people, and I am not sure my understanding is really } \\
\text { what it is, and I think fundamentally that is where we need } \\
\text { to start, is not only an education about the disease but also } \\
\text { an education about the treatments as well as okay what this } \\
\text { clinical trial is going to do." (Decliner-F) } \\
\text { "...but what happens is you get information overload.... I } \\
\text { think that one of things is, perhaps in your initial visit, that } \\
\text { information can be given, even before surgery. Because } \\
\text { you have an opportunity to process it, because I think after } \\
\text { the fact, I think the emotions of it all takes over, and you're } \\
\text { like having an out of body experience, you're not really } \\
\text { giving things proper weight. I know that's how it } \\
\text { was for me." (Decliner-F) }\end{array}$ \\
\hline
\end{tabular}

Subtheme:

communication; multifaceted information needs

Subtheme: economic feasibility and therapy side effects

Theme: intrapersonal perspectives

Theme: interpersonal influences information multiple times because being given too much information at once cause them to become overwhelmed. Receiving information at the right time, and not all at once, allowed participants to process and understand the information.

Two new findings emerged. First, participants recommended testimonials from patients who accepted and those who declined participation as an important information source. Participants also believed real-life narratives related to trial decision-making and participation would provide useful information.

Our data also revealed the importance of balancing information obtained from provider interactions, information gathered from additional sources, side effects, and how information regarding trials is communicated.

The decision to participate in a clinical trial was influenced by economic feasibility, fear of the side effects of therapy, or both. Some patients who declined participation mentioned a fear that being involved in a CCT would delay them from going back to work and have a negative financial impact on them. Others were concerned about clinical trial costs that would not be covered by insurance.

Economic issues and opportunities to address questions about the trial treatment and related concerns from providers and other sources were important in fuller understanding and greater confidence related to trial decision-making

When making CCT decisions, participants weighed their understanding of the need to participate in research studies as African-Americans against their awareness of historical mistreatment of minorities in research against slightly higher pressure to participate in studies due to minority status. The tension created by these large-scale concerns was described as complicating decisions and was often described in association with decisional regret regarding the ultimate trial-related decision.

While minority patients' mistrust of research and researchers has been well-documented in the literature, participants clearly perceived benefits related to trial participation. In spite of these reservations, participants described participation as a responsibility and obligation for minorities, and perceived enrollment invitations as an opportunity to potentially reduce or ameliorate health disparities

Although participants described trial decision-making as both personal and individual and were clear that process should
"I know there are other people that are already going through that, bring a couple of them even if it is good or bad, in to talk to you about this research." (Decliner-F)

"I think when they have trials like that, that should be offered before you go through your other stuff. You know, that would be your opportunity to make a difference. But they wait until you go through all this stuff, and then offer you a trial." (Acceptor-F)

"So now where, being in a recession I was thinking about trying to get back to work and so consequently I did not want to have to deal with anything that was gonna come in the way of my recuperation.... I would just rather go with the treatment I know is going to deal with the situation." (Decliner-F)

"But if it is something that you are involving, taking any kind of medication or they would need to know the side effects. The person would need to know,

'how is this going to affect my body, what are you trying to see?"” (Decliner-F)

"...but I did have some concern as to whether or not my supplemental insurance would cover what Medicare didn't." (Decliner-M)

"Black people, black men of my age cannot forget the Tuskegee experiment either... I do understand having somewhat of a scientific background, the need for research... So there is a profound need for us, let me include myself, to step up to the plate to give the scientists information about new initiatives, new drugs, new medicines, and so forth..." (Decliner-M)

"Because we do have a lot of distrust in our community; it is highly warranted, and so, consequently, when people approach you with different things, you do not know whether or not it is for real, and then also you do not know in fact the impact of affect it is going to have on you.... I think if not only on this issue but in general, we really focus more on the common good then we would be much better off. So this clinical trial is about the common good, so it is a good thing." (Decliner-F) 
Table 3 (continued)

Themes/subthemes Description $\quad$ Selected exemplar data (individual groups are de-identified)

Subtheme: spiritual guidance

Subtheme: decision partner(s)

Theme: decision processing/decision balance or imbalance

Theme: decision outcomes not be dictated for others, many included other people in the decision-making process. The exact nature of how decision-making process is shared and why decision partners discouraged participation was not a focus of these discussions and was planned for follow-up interviews. However, participants described the involvement of different levels of decision partners and supporters; each appeared to have different roles in the process. Neutral supporters (e.g., doctors, nurses, and pastors/clergy) provided a "listening ear," for reviewing the pros and cons of trial treatment. Family and friends often had stronger and more direct influence, particularly when participants were undecided.

Many participants turned to God and relied on their spirituality to help cope with their cancer diagnosis and clinical trial decision-making. Participants sought confirmation on the direction of their clinical trial decisions based on prayer and God. Regardless of the decision, spirituality helped participants feel more at ease with their decision and accept that their decision was correct

Previous research has focused on shared decision making between patients and providers [12]; In some situations, concordance between patient and family was present and provided reassurance that the decision made was appropriate.

Interpersonal experiences influenced satisfaction with these decisions. Decision satisfaction or, in some cases, regret was also influenced by concordance or discordance between participants and decision partners. Generally, participants who were satisfied with their decision reported high concordance between themselves and decision partners or other supporters.

For study participants, the decision-making process began with a provider or CT-team member offer to participate. Many were alone when approached with $\mathrm{CT}$ participation and required additional information to inform their decision. Financial feasibility in the face of insurance coverage and the potential benefits and burdens of being a minority patient were important factors in participants' decision-making. Whether they chose to participate or not, nearly all participants discussed balancing the pros and cons in collaboration with decision partners (spouses, nuclear family members) or praying for guidance.

Decision balance, a concept that has been noted in prior research, evolved as an a posteriori theme in this study [24]. Decisional balance is a core construct of the Transtheoretical Model of Behavior Change, measuring an individual's rating of the relative importance of the pros versus the cons of changing a specific behavior [25]. Participants' descriptions of a process of contemplating trial participation are

aligned with this construct.

Trial decision-making was challenging for all patients.

Participants who reported sufficient time to process trial information, positive interactions with clinical staff, and few trial side effects were more satisfied with their decision to participate and had less regret.
“The overriding influence, I think, didn't come from a medical practitioner. It came from my wife who was saying, no, no, no." (Decliner-M)

"Talking about the clinical trial, Dr. S and his team did a good job of explaining to me all of what went into that protocol..... However, I did decide after speaking to a couple family members, my wife included, that I would not participate in the clinical trial.” (Decliner-M)

"I prayed and God gave me confirmation because I was listening [to] a very conservative radio station, family radio... and they came on three times about clinical research trials and participating... I said, well, God you've given me the confirmation and I heard him three times. The thing was about to come on again. I said, nope, that's it. I got my answer." (Acceptor-F)

"When I was first diagnosed, I was terrified....and thanks to my higher power, which is God, that brought me more strength to understand. It didn't matter what direction I took, I just hold on to my faith, and just prayed a lot." (Decliner-F

"Me and my husband and my aunt, we all decided that it wasn't a good idea." (Decliner-F)

"Once they explained what was happening and I knew what I had to do... the side effects, and this and that... So it was all right. My sisters were behind me." (Acceptor-M)

"I read the information [consent form], it presented the pros and the cons. I felt that it wasn't going to harm me. I felt that it was going to help other people...I did discuss it with my mother and she said if you really want to, if it's not going to bring you any harm....." (Acceptor-F)

"I made a good decision, now I feel good, I feel good about my decision...Anything that I figure that can help the next person so they can deal with it and know what they are going to go through, that is really a plus, and it really is." (Acceptor-M)

"When they asked me about it, I heard all the ins and outs, they allowed me to question them.... I didn't jump to any hasty conclusions.... So you know, I think I made a good decision about it, I do." (Acceptor-F) 
Table 3 (continued)

Themes/subthemes Description

Selected exemplar data (individual groups are de-identified)

Subtheme: decisionrelated satisfaction

Subtheme: decisionrelated regret
Many participants valued advances in science and cancer. As a result, most acceptors acknowledged and expressed satisfaction surrounding their research contributions. Posttreatment, participants reflected on the perceived benefit in believing that

their personal experience could result in a greater good.

Some decliners expressed regret regarding what they perceived as a lost opportunity to increase cancer knowledge and address disparities.
"So, my contention was I am not ready to experiment, I want to get the cancer out of me and go forward. So, therefore I really was not open to it. So, what it was I had no clue, I dismissed it and moved on but in retrospect probably should have examined it further." (Decliner-F)

"Anything that I figure that can help the next person so they can deal with it and know what they are going to go through, that is really a plus....."

(Acceptor-M)

"I prayed on it and everything, and with the decision of my family, I know I made the right decision." (Decliner-F)

"...I really believe now that there should be some kind of other research... So now, in retrospect, I think I would like to try a clinical trial... it would help other women of color." (Decliner-F)

"I think I would have liked to have been better informed. It matters to me one way or the other about the clinical trial because I maybe would have said, 'dang, if I'm going to be that sick I mean, oh, well maybe I should have done the clinical trial.' Maybe I would have been able to help the next person or even myself..." (Decliner-F)
Trial Decision-making (Fig. 1), which depicted decisionmaking influences and outcomes. Broadly, information gathering, intrapersonal influences, and interpersonal influences were a part of decision processing. Further, participants' ability to achieve decision balance after decision processing appeared to impact whether they experienced satisfaction or regret related to their $\mathrm{CT}$ participation decision. Decision- making themes identified in this study (Fig. 1) were both similar and different to those previously identified in the literature. Similar to previous studies, common reasons for non-participation included medical distrust, lack of knowledge about clinical trials, negative interactions with healthcare providers, cost, transportation, time, and trial side effects [16]. In contrast, individuals who chose to participate often did so

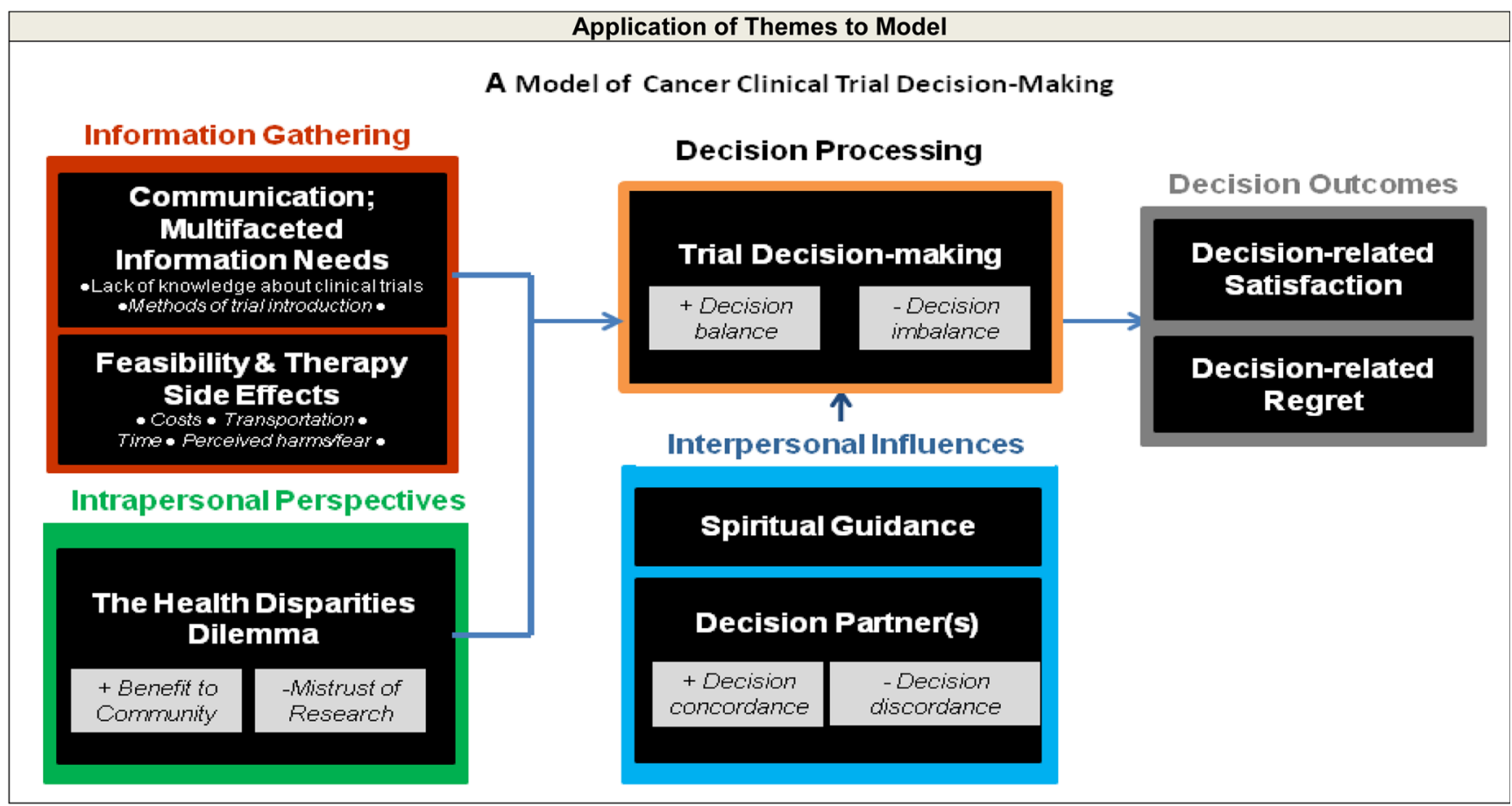

Fig. 1 Application of the themes/subthemes used to develop the Model of Cancer Clinical Trial Decision-making 
because they felt support from family and friends and/or spiritual motivation to participate [16]. Family and friends were trusted sources of clinical trial information and acted as decision partners [28]. Personal relevance of the trial was also important and affected participants' motivation to participate.

While clinicians and researchers often acknowledge healthcare disparities, we were intrigued to discover that the existence of such disparities was factored into participants' decision-making process. This theme, the health disparities dilemma, influenced and impacted the decision-making process for many participants (Fig. 1). As a part of the trial decision-making process, we believe the health disparities dilemma is a new contribution to the literature. As in other studies [29], participants who viewed trial participation as an opportunity to help others or ameliorate health and treatment disparities were more motivated to join clinical trials. Nevertheless, the health disparities dilemma was a source of tension and conflict surrounding trial decision-making and warrants additional exploration.

In our investigation of the decision-making process, we identified acceptors and decliners who experienced decision regret (Fig. 1). Specific reasons for decision regret, including those expressed by our participants, have not been well defined previously [19]. We found acceptors expressed regret when they felt they received inaccurate or inadequate information regarding what to expect from trial treatment. Decliners who initially feared the effect of cancer trials regretted not participating after they experienced the difficulties of standard cancer treatment (Fig. 1). Decision partners may facilitate decision satisfaction or contribute to regret, but there is little available literature describing the process by which they contribute to trial decision-making. Similarly, while altruism is wellestablished as a motivator for trial participation [29], perceived "loss" or regret related to altruism has not been emphasized. Because decision regret is closely associated with decision-making dissatisfaction [19], potential sources of regret warrant further investigation.

Our model (Fig. 1) integrates these concepts and may serve as a guide to develop strategies for recruitment of AfricanAmericans into clinical trials. Strategies to improve communication about the risks and benefits of trial participation and financial feasibility have been shown to facilitate the enrollment of African-Americans in clinical trials; [14] they may also help reduce decision regret among trial participants. Furthermore, because decision partners played an important role in participants' decisions to accept or decline trial participation, novel interventions that engage and support decision partners in assistive decision-making should be developed. Finally, the research team and approaches to clinical trial communication should be sensitive to potential participants' ethical concerns and internal conflicts specific to existing health disparities [14].
Limitations and Strengths

A major strength of this study is that it focused exclusively on individuals who had been offered participation in a cancer clinical trial, thereby permitting qualitative comparisons of acceptors vs. decliners of participation. However, some limitations should also be considered. Because of our sampling limitations, our findings cannot be generalized to AfricanAmericans, or to other underrepresented groups in clinical trials [30]. Although we achieved data saturation [31], model specificity may be limited. While we relied on patient selfreport of trial offerings, prior studies among middle-aged adults have shown high validity of self-reported data compared to registries of other measures such as cancer screening [23] and history [24, 25]. We attempted to offset potential social desirability bias by including both acceptors and decliners in the study. Finally, participants' narratives provided a helpful opportunity to corroborate whether patients were offered trial participation.

\section{Conclusion}

Our findings highlight the multiple and complex considerations surrounding trial participation from the perspectives of African-American cancer patients. Our model presents a framework for understanding the relationships between information gathering, intrapersonal perspectives, and interpersonal influences in trial decision-making. Our model may be used to further explore key factors that influence the process of decision-making and the achievement of positive decision outcomes rather than merely focusing on recruitment success. The model should be confirmed in additional AfricanAmerican populations and other underrepresented populations, including members of other minority groups. Our model may be used to guide the development of interventions to improve the process of trial decision-making and thereby improve decision outcomes.

Acknowledgments The authors thank Syed-Rafay Ahmed and Charlene Ndi for their contribution to the study/manuscript review. We also thank all study staff and our study participants. Support:Research reported in this publication was supported by the National Institute on Minority Health and Health Disparities of the National Institutes of Health under Award number U24MD006970. Dr. Wenzel is supported by the American Cancer Society MRSGT-09-152-01-CPPB. Dr. Wenzel and Ms. Mbah are supported in part by the Community Networks Program Center (CNPC)'s grant, U54CA153710. The content is solely the responsibility of the authors and does not necessarily represent the official views of the National Institutes of Health or the American Cancer Society. All procedures followed were in accordance with the ethical standards of the responsible committee on human experimentation (institutional and national) and with the Helsinki Declaration of 1975, as revised in 2000 (5). Informed consent was obtained from all patients for being included in the study. 
Conflict of Interest All authors declare that they have no conflict of interest.

\section{References}

1. Siegel R, Ward E, Brawley O, Jemal A. Cancer statistics. CA Cancer J Clin. 2011;61(4):212-36.

2. Adams-Campbell LL, Ahaghotu C, Gaskins M, et al. Enrollment of African Americans onto clinical treatment trials: study design barriers. J Clin Oncol. 2004;22(4):730-4.

3. Goss E, Lopez AM, Brown CL, Wollins DS, Brawley OW, Raghavan D. American society of clinical oncology policy statement: disparities in cancer care. J Clin Oncol. 2009;27(17):2881-5.

4. Baquet CR, Ellison GL, Mishra SI. Analysis of Maryland cancer patient participation in National Cancer Institute-supported cancer treatment clinical trials. J Health Care Poor Underserved. 2009;20(2 Suppl):120-34.

5. Evans KR, Lewis MJ, Hudson SV. The role of health literacy on African American and Hispanic/Latino perspectives on cancer clinical trials. J Cancer Educ. 2012;27(2):299-305.

6. Lai GY, Gary TL, Tilburt J, et al. Effectiveness of strategies to recruit underrepresented populations into cancer clinical trials. Clin Trials. 2006;3(2):133-41.

7. Djulbegovic B, Kumar A, Soares HP, et al. Treatment success in cancer: new cancer treatment successes identified in phase 3 randomized controlled trials conducted by the National Cancer Institutesponsored cooperative oncology groups, 1955 to 2006. Arch Intern Med. 2008;168(6):632-42.

8. Braunholtz DA, Edwards SJ, Lilford RJ. Are randomized clinical trials good for us (in the short term)? Evidence for a "trial effect". J Clin Epidemiol. 2001;54(3):217-24.

9. Chow CJ, Habermann EB, Abraham A, et al. Does enrollment in cancer trials improve survival? J Am Coll Surg. 2013;216(4):774-80.

10. Tanai C, Nokihara H, Yamamoto S, et al. Characteristics and outcomes of patients with advanced non-small-cell lung cancer who declined to participate in randomised clinical chemotherapy trials. Br J Cancer. 2009;100(7):1037-42.

11. Du W, Gadgeel SM, Simon MS. Predictors of enrollment in lung cancer clinical trials. Cancer. 2006;106(2):420-5.

12. Colon-Otero G, Smallridge RC, Solberg Jr LA, et al. Disparities in participation in cancer clinical trials in the United States: a symptom of a healthcare system in crisis. Cancer. 2008;112(3):447-54.

13. Ford JG, Howerton M. The science of recruiting minority populations to screening trials. Clin Trials. 2004;1(4):341-2.

14. Advani AS, Atkeson B, Brown CL, et al. Barriers to the participation of African-American patients with cancer in clinical trials: a pilot study. Cancer. 2003;97(6):1499-506.

15. Ford JG, Howerton MW, Lai GY, et al. Barriers to recruiting underrepresented populations to cancer clinical trials: a systematic review. Cancer. 2008;112(2):228-42.
16. Rivers D, August EM, Sehovic I, Lee Green B, Quinn GP. A systematic review of the factors influencing African Americans' participation in cancer clinical trials. Contemp Clin Trials. 2013;35(2):13-32.

17. Cox K, McGarry J. Why patients don't take part in cancer clinical trials: an overview of the literature. Eur J Cancer Care (Engl). 2003;12(2):114-22.

18. COX K, Avis M. Ethical and practical problems of early anti-cancer drug trials: a review of the literature. European Journal of Cancer Care. 1996;5(2):90-5.

19. Stryker JE, Wray RJ, Emmons KM, Winer E, Demetri G. Understanding the decisions of cancer clinical trial participants to enter research studies: factors associated with informed consent, patient satisfaction, and decisional regret. Patient Educ Couns. 2006;63(1-2):104-9.

20. Fischer C. Qualitative research methods for psychologists: introduction through empirical studies. Burlington, MA: Academic; 2006.

21. MacDougall C, Fudge E. Planning and recruiting the sample for focus groups and in-depth interviews. Qual Health Res. 2001;11(1): $117-26$.

22. Morgan D, Kruger RA. The focus group kit. Thousand Oaks, CA: Sage Publications; 1998.

23. Baier M, Calonge N, Cutter G, et al. Validity of self-reported colorectal cancer screening behavior. Cancer Epidemiol Biomarkers Prev. 2000;9(2):229-32.

24. Bergmann MM, Calle EE, Mervis CA, Miracle-McMahill HL, Thun MJ, Heath CW. Validity of self-reported cancers in a prospective cohort study in comparison with data from state cancer registries. Am J Epidemiol. 1998;147(6):556-62.

25. Stavrou E, Vajdic CM, Loxton D, Pearson SA. The validity of selfreported cancer diagnoses and factors associated with accurate reporting in a cohort of older Australian women. Cancer Epidemiol Biomarkers Prev. 2011;35(6):e75-80.

26. Steeves RH, Kahn DL, Cohen MZ. Technical notes asking substantive theory questions of naturalistically derived data. West J Nurs Res. 1996;18:209-12.

27. Wenzel J, Jones RA, Klimmek R, et al. Cancer support and resource needs among African American older adults. Clin J Oncol Nurs. 2012;16(4):372-7.

28. Phillips CA, Green HJ, Morrissey SA. Cognitive and affective correlates of decisional balance regarding screening mammography in older women. Psychol Health Med. 2012;17(2):16475.

29. Flynn KE, Weinfurt KP, Seils DM, et al. Decisional conflict among patients who accept or decline participation in phase I oncology studies. J Empir Res Hum Res Ethics. 2008;3(3):6977.

30. Denzin NK, Lincoln YS. Handbook of qualitative research. 2nd ed. Thousand Oaks, CA: Sage Publications; 2000.

31. Morse JM, Penrod J, Hupcey JE. Qualitative outcome analysis: evaluating nursing interventions for complex clinical phenomena. $\mathrm{J}$ Nurs Scholarsh. 2000;32(2):125-30. 